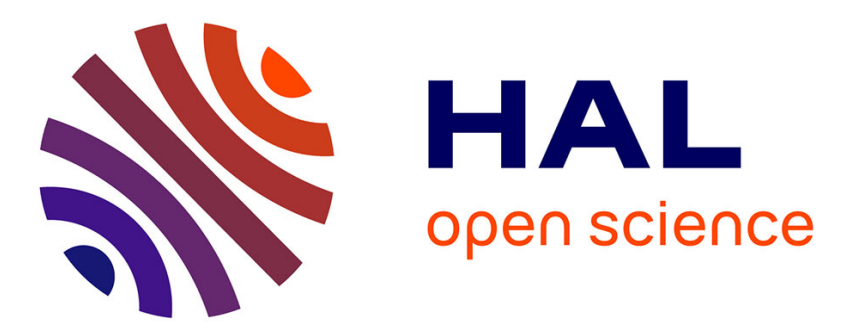

\title{
Consensus value estimates in time-varying and directed networks
}

\author{
Samuel Martin, Irinel-Constantin Morarescu, Dragan Nesic
}

\section{To cite this version:}

Samuel Martin, Irinel-Constantin Morarescu, Dragan Nesic. Consensus value estimates in time-varying and directed networks. 56th IEEE Conference on Decision and Control, CDC 2017, Dec 2017, Melbourne, Australia. hal-01653084

\section{HAL Id: hal-01653084 https://hal.science/hal-01653084}

Submitted on 1 Dec 2017

HAL is a multi-disciplinary open access archive for the deposit and dissemination of scientific research documents, whether they are published or not. The documents may come from teaching and research institutions in France or abroad, or from public or private research centers.
L'archive ouverte pluridisciplinaire HAL, est destinée au dépôt et à la diffusion de documents scientifiques de niveau recherche, publiés ou non, émanant des établissements d'enseignement et de recherche français ou étrangers, des laboratoires publics ou privés. 


\title{
Consensus value estimates in time-varying and directed networks
}

\author{
Samuel Martin*, Irinel-Constantin Morărescu*, Dragan Nešić\#
}

\begin{abstract}
The paper provides consensus value estimates in multi-agent systems with directed and time-varying interaction networks. First, we prove general results regarding the asymptotic consensus value which is obtained as a convex combination of the initial states. It is shown that the cutbalance assumption guarantees a strictly positive lower bound on the convex combination components. This means that each agent plays a non vanishing role in the asymptotic consensus value. Second, we analyze the case where interaction weights vary uniformly over time. Finally, we study the effect of timevanishing perturbations on the systems with uniform variation of the interaction weights. We show that the convex combination components vary smoothly with the perturbation under smooth and sufficiently fast vanishing perturbations. Moreover, we show that in this case, these components reach a limit when time goes to infinity. We also provide an example where this limit does not exist when the perturbation does not respect the fast vanishing assumption although the system itself converges to a consensus. Some numerical examples illustrate our results.
\end{abstract}

\section{INTRODUCTION}

Analysis and control of multi-agent systems have attracted a lot of attention during the last decade. Due to decentralized behavior and control of subsystems (agents), the multi-agent framework has applications in a wide variety of domains such as robotic teams, energy and telecommunication networks, opinion dynamics in social networks, analysis of biological networks, etc. The coherent behavior of the agents in such systems is often described in terms of consensus, i.e., the agents have to reach agreement in some variables of interest.

Consensus and synchronization problems have been studied in different contexts : linear or nonlinear agent dynamics [1], [2], [3], fixed or time-varying interconnection topologies [4], [5], fixed or time-varying weights of the agents influence [6], [7], directed or undirected and synchronous or asynchronous information exchange [8], [9], [10].

Beside the guarantees of reaching consensus, an important related issue is the estimation of the asymptotic consensus value in terms of initial condition and interaction network. For linear dynamics, as it will be made explicit in the sequel, the asymptotic consensus value is always a convex combination of the initial state, and the components of the convex combination depend on the interaction network

The work of S. Martin and I.-C. Morărescu was supported by project PEPS MoMIS MADRES and PEPS INS2I CONAS funded by the CNRS and project Computation Aware Control Systems (COMPACS), ANR-13BS03-004 funded by ANR. The work of D. Nešić was supported by the Australian Research Council under the Discovery Project DP1094326.

* Université de Lorraine, CRAN, UMR 7039, and CNRS, CRAN, UMR 7039, 2, Avenue de la Forêt de Haye, 54500, Vandœuvre, France. (samuel.martin,constantin.morarescu)@univ-lorraine.fr.

\# Department of Electrical and Electronic Engineering, The University of Melbourne, Parkville, 3010 VIC, Australia. dnesic@unimelb.edu.au over time. The characterization of the asymptotic consensus value has been solved for some special cases: when the interaction network is balanced, i.e., the adjacency matrix is doubly stochastic, the consensus is always the average of the initial states [4]. For directed but fixed interactions, the convex combination of initial states corresponds to the left normalized eigenvector of the Laplacian matrix (see for instance [4]). The consensus value for some particular linear impulsive systems was characterized in [11]. However, characterizing the asymptotic consensus value in the general case of directed time-varying interaction network is a hard problem.

The paper presents several contributions on consensus of multi-agent systems with linear continuous-time dynamics under directed and time-varying interaction weights. On the one hand it provides a characterization and an estimation of the asymptotic consensus value. It is shown that the asymptotic consensus value is a convex combination of the current state and this convex combination can be expressed in terms of the fundamental (state transition) matrix. Moreover, it is proven that when the interaction network satisfies the cut-balance property, the components of the convex combination are non-trivially lower bounded. In other words, each single agent is responsible for part of the asymptotic consensus value. On the other hand, it considers the problem of approximation of consensus value for perturbed dynamics. First, we show that in the special case where the interaction weights vary uniformly throughout the network the asymptotic consensus value can be easily determined. Next, we focus on the approximation of the consensus value of perturbed dynamics. Precisely, we consider the case of uniform variation of the interaction in presence of an additive perturbation of magnitude characterized by a parameter $\varepsilon$. We show that the convex combination components vary smoothly with the parameter $\varepsilon$ when the perturbations are smooth and vanish sufficiently fast in time. Moreover, we show that in this case, the convex combination components reach a limit when time goes to infinity. This is a non trivial result since we also provide an example where this limit does not exist when the perturbation does not respect the fast vanishing assumption although the system itself converges to a consensus.

Notation. The following notation will be used throughout the paper. The sets of non-negative integers, real and nonnegative real numbers are denoted by $\mathbb{N}, \mathbb{R}$ and $\mathbb{R}_{+}$, respectively. For a vector $v$ and a matrix $A$ we denote by $\|v\|_{\infty}$ and $\|A\|_{\infty}$ their infinity norms. We denote by $\mathcal{L}_{1}$ the space of integrable function on $\mathbb{R}_{+}$. For a matrix-valued function of time $M$ in $\mathcal{L}_{1}$, we denote $\|M\|=\int_{t_{0}}^{+\infty}\|M(s)\|_{\infty} d s$ where 
$t_{0}$ is a fixed initial time. The transpose of a matrix $A$ is denoted by $A^{\top}$. By $I_{k}$ we denote the $k \times k$ identity matrix. $\mathbf{1}_{k}$ and $\mathbf{0}_{k}$ are the column vectors of size $k$ having all the components equal 1 and 0 , respectively. Vector $e_{i}$ denotes the $i$-th canonical vector in $\mathbb{R}^{n}$, i.e., the vector of all zeros but one 1 for its $i$-th coefficient, for some given $i \in \mathcal{N}$.

A non trivial subset $S$ of a set $C$, denoted as $S \sqsubset C$, is a non-empty set with $S \subsetneq C$. A directed path of length $p$ in a given directed graph $\mathcal{G}=(\mathcal{N}, \mathcal{E})$ is a union of directed edges $\bigcup_{k=1}^{p}\left(i_{k}, j_{k}\right)$ such that $i_{k+1}=j_{k}, \forall k \in\{1, \ldots, p-1\}$. The node $j$ is connected to node $i$ in a directed graph $\mathcal{G}=(\mathcal{N}, \mathcal{E})$ if there exists at least one directed path in $\mathcal{G}$ from $i$ to $j$.

\section{PREliminaries}

\section{A. Model statement}

Let $\mathcal{N} \triangleq\{1, \ldots, n\}$ be a set of $n \geq 2$ agents. By abuse of notation we denote both an agent and its index by the same symbol $i \in \mathcal{N}$. Each agent is characterized by a scalar state $x_{i} \in \mathbb{R}, \forall i \in \mathcal{N}$ that evolves according to the following consensus system

$$
\begin{aligned}
& \dot{x}_{i}\left(t, \varepsilon, t_{0}\right)=\sum_{j=1}^{n} a_{i j}(t, \varepsilon)\left(x_{j}\left(t, \varepsilon, t_{0}\right)-x_{i}\left(t, \varepsilon, t_{0}\right)\right), \\
& x_{i}\left(t_{0}, \varepsilon, t_{0}\right)=x_{i, 0},
\end{aligned}
$$

where, $\varepsilon \in I$ with $I \subset \mathbb{R}_{+}$a fixed interval. Interval $I$ may or may not include 0 . We assume that interval $I$ has a finite suppremum $\varepsilon^{*}$. The functions $a_{i j}: \mathbb{R}_{+} \times I \rightarrow \mathbb{R}_{+}$ are twice continuously differentiable and uniformly upperbounded functions in both variables, representing the interaction weights. We denote the supremum, for $\varepsilon \in I$, as

$$
\bar{a}(\varepsilon)=\sup _{i, j \in \mathcal{N}, t \geq 0} a_{i j}(t, \varepsilon) .
$$

Let $x\left(t, \varepsilon, t_{0}\right)=\left(x_{1}\left(t, \varepsilon, t_{0}\right), \ldots, x_{n}\left(t, \varepsilon, t_{0}\right)\right)^{\top} \in \mathbb{R}^{n}$ be the overall state of the network collecting the states of all the agents. Existence, uniqueness and smoothness of the solution $x$ is given in the following lemma.

Lemma 1: Let the initial time $t_{0}$ and initial condition $x\left(t_{0}, \varepsilon, t_{0}\right)=x_{0}$, independent of $\varepsilon$, be given. Under the smoothness and boundedness assumptions on $a_{i j}(t, \varepsilon)$, for any fixed $\varepsilon$ there exists a unique function $x\left(\cdot, \varepsilon, t_{0}\right)$ : $\left[t_{0}, \infty\right) \rightarrow \mathbb{R}^{n}$ whose components satisfy equation (1) for all $t \in\left[t_{0}, \infty\right)$. Moreover, this function is bounded and twice continuously differentiable with regards to both $t$ and $\varepsilon$ and its first and second partial derivatives are continuous for all $t \geq t_{0}$ and $\varepsilon \in I$.

Proof: The existence and uniqueness hold true even for more general classes of weight functions and can be found in [12]. The fact that it is bounded was proven in [6]. The smoothness result with regards to time $t$ and parameter $\varepsilon$ comes from Theorem p285 in [13].

We call the solution of system (9) the trajectory of the overall system and we denote it by $x\left(\cdot, \varepsilon, t_{0}\right)$ to point out its dependence on the parameters $\varepsilon$ and $t_{0}$. We say the trajectory asymptotically reaches a consensus when there exists a common agreement value $\alpha\left(t_{0}, \varepsilon\right) \in \mathbb{R}$ such that

$$
\lim _{t \rightarrow+\infty} x_{i}\left(t, \varepsilon, t_{0}\right)=\alpha\left(t_{0}, \varepsilon\right), \forall i \in \mathcal{N} .
$$

When convergence to consensus occurs, we denote

$$
x^{*}\left(t_{0}, \varepsilon\right)=\lim _{t \rightarrow+\infty} x\left(t, \varepsilon, t_{0}\right)=\alpha\left(t_{0}, \varepsilon\right) \mathbf{1}_{n} .
$$

In the sequel, we are interested in estimating both the consensus value $\alpha\left(t_{0}, \varepsilon\right)$ and the convergence speed toward this value.

For any $\varepsilon \in I$ we denote $A(t, \varepsilon)=\left[a_{i j}(t, \varepsilon)\right]_{i j}$ the adjacency matrix of communication weights at time $t$, $D(t, \varepsilon)=\operatorname{diag}\left(d_{i i}(t, \varepsilon)\right)$ with $d_{i i}(t, \varepsilon)=\sum_{j \in \mathcal{N}} a_{i j}(t, \varepsilon)$, and $L(t, \varepsilon)=D(t, \varepsilon)-A(t, \varepsilon)$ its associated Laplacian matrix.

\section{B. Framework assumptions}

In the following let us introduce some notation and the main hypotheses of this work. We denote

$$
c^{I}(t, \varepsilon)=\min _{S \sqsubset \mathcal{N}} \sum_{i \in S, j \notin S} a_{i j}(t, \varepsilon),
$$

which is known as the edge connectivity [14].

Assumption 1: The interaction weights satisfy the two following properties :

- $c^{I}(t, \varepsilon)>0, \forall t \geq t_{0}$ and $\int_{t_{0}}^{\infty} c^{I}(t, \varepsilon) d s=+\infty$.

- cut-balance: there exists $K_{I}(\varepsilon) \geq 1$ such that for all non trivial subsets $S \sqsubset \mathcal{N}, t \geq t_{0}$ and $\varepsilon \in I$,

$$
\sum_{i \in S, j \notin S} a_{i j}(t, \varepsilon) \leq K_{I}(\varepsilon) \cdot \sum_{i \in S, j \notin S} a_{j i}(t, \varepsilon),
$$

Remark 1: Notice that Assumption 1 and the upper bound $\bar{a}(\varepsilon)$ on the interaction weights defined in equation (2) does not imply a uniform lower bound on the non-zero interaction weights. In fact, to satisfy the lower bound on the edgeconnectivity given in the first point in Assumption 1, some weights $a_{i j}(t, \varepsilon)$ may converge to 0 as long as this is compensated by some other stronger weights $a_{i k}(t, \varepsilon)$.

The following result states that Assumption 1 ensures that asymptotic consensus is reached.

Proposition 1: Suppose that Assumption 1 holds. Then, for any fixed $\varepsilon \in I$, and initial time $t_{0}$, the trajectory of system (1) reaches consensus (whose value generally depends on $t_{0}$ and $\varepsilon$ ).

Proof: For any fixed $\varepsilon \in I$, the second point in Assumptions 1 guarantees cut-balance of the communication weights. Moreover, the divergence imposed on the integral of $c^{I}(t, \varepsilon)$ by the first point in Assumption 1 guarantees that there exists a strongly connected graph $\mathcal{G}=(\mathcal{N}, \mathcal{E})$ such that $\int_{t_{0}}^{\infty} a_{i j}(s, \varepsilon) d s=\infty, \forall(j, i) \in \mathcal{E}$ and following [7, Proposition 4] one obtains the convergence toward consensus.

Remark 2: Proposition 1 applies in the more general setting of integral equations with non smooth $a_{i j}$ (see [6]) but in the present study, we use only twice continuously differentiable $a_{i j}$ due to the perturbation analysis that is presented in the Section IV.

From Proposition 1, it is known that the trajectory of system (1) will converge to consensus. It remains to characterize the consensus value $\alpha\left(t_{0}, \varepsilon\right)$. The analysis is carried out for general time-varying, non-symmetric communication weights. 


\section{ANALYSIS OF PARAMETRIC TIME-VARYING CONSENSUS SYSTEMS}

The aim of this section is to study the behavior of the time-varying consensus system (1) parametrized by $\varepsilon \in I$. Precisely, we give a characterization of the consensus value as a convex combination of the current state at any time $t$. In general, we show that non-trivial bounds can be obtained on the components of the convex combination provided that the interaction weights satisfiy some reciprocity condition. We point out that in some special cases the convex combination is time-invariant and easy to compute.

\section{A. Consensus value}

Using the matrix notation, system (1) can be represented as

$$
\dot{x}\left(t, \varepsilon, t_{0}\right)=-L(t, \varepsilon) x\left(t, \varepsilon, t_{0}\right), \quad x\left(t_{0}, \varepsilon, t_{0}\right)=x_{0} .
$$

For all $\varepsilon \in I$ we define, for all $t, t_{1} \geq t_{0}$ with $t \geq t_{1}$, the fundamental matrix $\Phi\left(t, \varepsilon, t_{1}\right)$ of system (5) such that

$$
x\left(t, \varepsilon, t_{0}\right)=\Phi\left(t, \varepsilon, t_{1}\right) x\left(t_{1}, \varepsilon, t_{0}\right) .
$$

It is important to define $\Phi$ not only for $t_{1}=t_{0}$ since in the sequel, we will need to study $\lim _{t_{1} \rightarrow \infty} \lim _{t \rightarrow \infty} \Phi\left(t, \varepsilon, t_{1}\right)$. By definition, the fundamental matrix $\Phi\left(t, \varepsilon, t_{1}\right)$ is independent of the initial state $x_{0}$ and for all $t_{1} \geq t_{0}$ it is solution of the following system :

$$
\left\{\begin{array}{l}
\frac{\partial \Phi}{\partial t}\left(t, \varepsilon, t_{1}\right)=-L(t, \varepsilon) \Phi\left(t, \varepsilon, t_{1}\right), \\
\Phi\left(t_{1}, \varepsilon, t_{1}\right)=I .
\end{array}\right.
$$

Lemma 2: The fundamental matrix $\Phi\left(t, \varepsilon, t_{1}\right)$ associated to the general consensus system (1) is twice continuously differentiable with regards to $t$ and $\varepsilon$ and its partial derivative with regards to $\varepsilon$ evolves according to dynamics

$$
\frac{\partial}{\partial t} \frac{\partial \Phi}{\partial \varepsilon}\left(t, \varepsilon, t_{1}\right)=-L(t, \varepsilon) \frac{\partial \Phi}{\partial \varepsilon}\left(t, \varepsilon, t_{1}\right)-\frac{\partial L}{\partial \varepsilon}(t, \varepsilon) \Phi\left(t, \varepsilon, t_{1}\right) .
$$

Furthermore, if for all $\varepsilon \in I, \frac{\partial L}{\partial \varepsilon}(t, \varepsilon)$ is in $\mathcal{L}_{1}$ and

$$
d_{L}(\varepsilon) \triangleq \int_{t_{0}}^{\infty}\left\|\frac{\partial L}{\partial \varepsilon}(t, \varepsilon)\right\|_{\infty} d t<+\infty,
$$

then $\frac{\partial \Phi}{\partial \varepsilon}(t, \varepsilon)$ is bounded uniformly in time $t \geq t_{1}$ :

$$
\sup _{t \geq t_{1}}\left\|\frac{\partial \Phi}{\partial \varepsilon}\left(t, \varepsilon, t_{1}\right)\right\|_{\infty} \leq n d_{L}(\varepsilon) .
$$

Proof: See Appendix A in [15].

As a direct consequence of Lemma 6 in [16], for all $t_{1}, t \geq$ $t_{0}$ with $t \geq t_{1}$, for any $i, j \in \mathcal{N}$, weight $\Phi_{i j}\left(t, \varepsilon, t_{1}\right)$ is nonnegative and

$$
\sum_{j \in \mathcal{N}} \Phi_{i j}\left(t, \varepsilon, t_{1}\right)=1
$$

Lemma 3: For any $t_{1} \geq t_{0}$ and $\varepsilon \in I$, matrix $\Phi\left(t, \varepsilon, t_{1}\right)$ has a limit when $t \rightarrow \infty$, which we denote by

$$
\Phi^{*}\left(t_{1}, \varepsilon\right)=\lim _{t \rightarrow \infty} \Phi\left(t, \varepsilon, t_{1}\right) .
$$

Moreover, there exists $q\left(t_{1}, \varepsilon\right) \in \mathbb{R}^{n}$ such that

$$
\Phi^{*}\left(t_{1}, \varepsilon\right)=\mathbf{1}_{n} \cdot\left(q\left(t_{1}, \varepsilon\right)\right)^{T} \text {. }
$$

Similarly to $\Phi\left(t, \varepsilon, t_{1}\right)$, the vector $q\left(t_{1}, \varepsilon\right)$ is independent of the state $x\left(t_{1}, \varepsilon, t_{0}\right)$.

Proof: See [15].

The vector $q(t, \varepsilon)$ plays a major role in the rest of the study, in particular it relates the final consensus value $\alpha\left(t_{0}, \varepsilon\right)$ defined in equation (3) to the current states $x\left(t, \varepsilon, t_{0}\right)$ as given in the next lemma.

Lemma 4: The coefficients $q_{i}(t, \varepsilon)$ are non-negative for all $i \in \mathcal{N}$ and $t \geq t_{0}$, and sum up to one :

$$
q(t, \varepsilon)^{T} \mathbf{1}_{n}=1
$$

Furthermore, quantity $q(t, \varepsilon)^{T} x\left(t, \varepsilon, t_{0}\right)$ is invariant in time and defines the consensus value, i.e.,

$$
\forall \varepsilon \in I, \forall t \geq t_{0}, q(t, \varepsilon)^{T} x\left(t, \varepsilon, t_{0}\right)=\alpha\left(t_{0}, \varepsilon\right) .
$$

Proof: See [15].

Lemma 4 shows that the consensus value $\alpha\left(t_{0}, \varepsilon\right)$ is a convex combination of the current states $x_{i}\left(t, \varepsilon, t_{0}\right)$ weighted by the coefficients in vector $q(t, \varepsilon)$. Accordingly, knowing bounds on coefficients $q_{i}(t, \varepsilon)$ would result in estimates for the consensus value $\alpha\left(t_{0}, \varepsilon\right)$. We provide such bounds in the next theorem.

Theorem 1: Under Assumption 1, the components $q_{i}(t, \varepsilon)$ are positive uniformly bounded i.e., for all $t \geq t_{0}$ and $i \in$ $\{1, \ldots, n\}, n \geq 2$ one has

$$
q_{i}(t, \varepsilon) \in\left[q_{\min }(\varepsilon), q_{\max }(\varepsilon)\right] \in(0,1),
$$

with

$$
q_{\min }(\varepsilon)=\left(\frac{e^{-K_{I}(\varepsilon)}}{n}\right)^{n-1}, q_{\max }(\varepsilon)=1-(n-1) q_{\min }(\varepsilon) \text {. }
$$

Proof: See Appendix C in [15].

One can check that $q_{\min }(\varepsilon) \in\left(0, \frac{1}{n}\right)$ and $q_{\min }(\varepsilon) \leq$ $q_{\max }(\varepsilon)$.

Remark 3: Theorem 1 can be interpreted as follows: when the interaction network satisfies the cut-balance property, at any time, the current state of each single agents is responsible for part of the asymptotic value. This is clearly not trivial since for an interaction network with a tree topology, it can be shown that the consensus value is the initial state of the root, no matter the initial state of the other agents. Note that Theorem 1 could have been stated on consensus systems with no dependance on a parameter $\varepsilon$, but we kept this notation to remain consistent over the paper.

Corollary 1: Suppose Assumption 1 holds. Then, the final consensus value of (1) is within the following bounds:

$$
\alpha\left(t_{0}, \varepsilon\right) \in\left[q_{\min }(\varepsilon) \cdot \min _{i \in \mathcal{N}} x_{i, 0}, q_{\max }(\varepsilon) \cdot \max _{i \in \mathcal{N}} x_{i, 0}\right]
$$

where $q_{\min }(\varepsilon)$ and $q_{\max }(\varepsilon)$ are defined in Theorem 1 .

Remark 4: Two other important properties of vector $q$ can be stated. One is smoothness with regards to time $t$ and the other is smoothness with regards to parameter $\varepsilon$ under some additional conditions on Laplacian matrix $L(t, \varepsilon)$. However, due to space limitation we limit our study to the second property. 


\section{B. Consensus under uniformly varying interaction weights}

In this part we consider a special case of uniform variation of the interaction weights throughout the network. Here, we assume that interval $I$ reduces to the singleton $\{0\}$. This case will correspond in Section IV to taking the perturbation parameter $\varepsilon=0$. As a consequence, we momentarily drop the $\varepsilon$ dependency of weights $a_{i j}$ and Laplacian $L$. The uniform variation of the interaction weights corresponds to assuming that for all couples of agents $(i, j)$ and $(k, h)$,

$$
\frac{a_{i j}(t)}{a_{k h}(t)}=\frac{a_{i j}\left(t_{0}\right)}{a_{k h}\left(t_{0}\right)} \text { whenever } a_{h k}\left(t_{0}\right) \neq 0,
$$

which is imposed by the following assumption.

Assumption 2: The interconnection network at instant $t_{0}$ is strongly connected and there exists a twice continuously differentiable, strictly positive and uniformly non-trivially lower and upper bounded function $l(t)$ with $l\left(t_{0}\right)=1$ and fixed initial interaction weights $b_{i j}$ so that

$$
\forall t \geq t_{0}, \forall i, j \in \mathcal{N}, a_{i j}(t)=l(t) b_{i j} .
$$

Then, matrix $L(t)=l(t) L_{0}$ where $L_{0}$ is the Laplacian matrix associated to adjacency matrix $\left[b_{i j}\right]_{i j}$.

Lemma 5: Assumption 2 implies Assumption 1 for the special case $I=\{0\}$. Consequently all the results proven under Assumption 1 hold true under Assumption 2.

Proof: Notice that a particular case of uniformly varying interaction weights is when they are constant, this corresponds to $l(t)=1, \forall t \geq t_{0}$. Then, it can be shown easily that any strongly connected interaction network verifies Assumption 1 : the integral in the first item increases linearly since there is a uniform lower bound $c^{I}(t, 0) \geq \underline{c}>0$, which depends on the interaction network at time $t_{0}$ and $K_{I}(0)$ is also computed at time $t_{0}$.

In the general case, we introduce the following notation

$$
\bar{l}=\sup _{t \geq t_{0}} l(t), \quad \underline{l}=\inf _{t \geq t_{0}} l(t)>0,
$$

and

$$
\bar{b}=\max _{i, j \in \mathcal{N}} b_{i j}, \quad \underline{b}=\min _{(i, j \in \mathcal{E})} b_{i j}>0 .
$$

Then, the weighted supremum defined in equation (2) is $\bar{a}(0)=\bar{l} \cdot \bar{b}$. Also, for the first point of Assumption 1, we have $c^{I}(t, 0) \geq \underline{l} c^{I}\left(t_{0}, 0\right)$ which allows computing $\underline{c}$ with respect to the initial condition and $\underline{l}$. Finally, regarding the second point of Assumption 1, $K_{I}(0)$ only depends on the ratio between the interaction weights and is therefore independent of $l(t)$ but only on the $b_{i j}$ which are constant and form a strongly connected graph.

Under Assumption 2, system (1) becomes

$$
\dot{x}\left(t, t_{0}\right)=-l(t) L_{0} x\left(t, t_{0}\right) .
$$

Notice that since $l(t) L_{0}$ and $l\left(t_{1}\right) L_{0}$ commutes for any $t, t_{1} \geq t_{0}$, the fundamental matrix for system (11) is

$$
\Phi\left(t, 0, t_{1}\right)=e^{-\tau\left(t, t_{1}\right) L_{0}},
$$

where $\tau\left(t, t_{1}\right)=\int_{t_{1}}^{t} l(s) d s$. Denote $p_{0}$ the left eigenvector of $L_{0}$ associated to eigenvalue 0 such that $p_{0}^{T} \mathbf{1}_{n}=1$.
In the case where the interaction weights vary uniformly, we show that Theorem 1 provides bounds on the coefficients of the Laplacian left eigenvector $p_{0}$.

Proposition 2: Suppose that Assumption 2 is satisfied. Then, we have

$$
\lim _{t \rightarrow \infty} e^{-\tau\left(t, t_{1}\right) L_{0}}=\mathbf{1}_{n} p_{0}^{T},
$$

and the left eigenvector $p_{0}$ of $L_{0}$ satisfies for all $t_{1} \geq t_{0}$,

$$
q\left(t_{1}, 0\right)=p_{0} \quad \text { and } \quad p_{0 i} \in\left[q_{\min }(0), q_{\max }(0)\right],
$$

where $q_{\min }(0)$ and $q_{\max }(0)$ are defined in Theorem 1 .

Proof: Under Assumption 2, the product $p_{0}{ }^{T} x\left(t, t_{0}\right)$ is time invariant :

$$
\begin{aligned}
\frac{d\left(p_{0}{ }^{T} x\right)}{d t}\left(t, 0, t_{0}\right) & =p_{0}^{T} \dot{x}\left(t, 0, t_{0}\right) \\
& =-l(t) p_{0}{ }^{T} L_{0} x\left(t, t_{0}\right)=0
\end{aligned}
$$

As a consequence, since by Lemma 5 and Proposition 1, trajectory $x\left(t, 0, t_{0}\right)$ converges, we have for all $t_{1} \geq t_{0}$,

$$
\begin{aligned}
p_{0}^{T} x\left(t_{1}, 0, t_{0}\right) & =\lim _{t \rightarrow \infty} p_{0}^{T} x\left(t, 0, t_{0}\right)=p_{0}^{T} x^{*}\left(t_{0}, 0\right) \\
& =\alpha\left(t_{0}, 0\right) p_{0}{ }^{T} \mathbf{1}_{n}=\alpha\left(t_{0}, 0\right) .
\end{aligned}
$$

Moreover, we also have according to Lemma 4,

$$
q\left(t_{1}, 0\right)^{T} x\left(t_{1}, 0, t_{0}\right)=\alpha\left(t_{0}, 0\right) .
$$

Since the two previous equalities holds for any initial conditions, and in particular so that $x\left(t_{1}, 0, t_{0}\right)=e_{i}$ (see the proof of Lemma 3 for a detailed explanation), we see that for all $t_{1} \geq t_{0}$,

$$
q\left(t_{1}, 0\right)=p_{0}, \forall t_{1} \geq t_{0} .
$$

The first equality comes from the definition of $q$ in function of the fundamental matrix $\Phi$ and from equation (12). Then, as a direct corollary of Theorem 1, we obtain the expected bound.

\section{Consensus VAlue ESTIMATE UNDER PERTURBED COMMUNICATION}

\section{A. Main result}

In this section, we explore how the consensus value evolves under perturbation of the dynamics (11) with uniformly varying weights. We focus on dynamics (1) where the interaction weights are of the form

$$
a_{i j}(t, \varepsilon)=l(t) b_{i j}+\varepsilon m_{i j}(t),
$$

where $\varepsilon \in I$ is a small perturbation parameter. Here, we assume either $I=\left[0, \varepsilon^{*}\right)$ or $I=\left[0, \varepsilon^{*}\right]$ with $\varepsilon^{*}>0$. As in Assumption 2, functions $l(t)$ and $m(t)$ are twice continuously differentiable and uniformly upper-bounded with $l(0)=1$ and $b_{i j}$ are fixed constant weights. To make sure that the $a_{i j}(t, \varepsilon)$ correspond to the components of a weighted adjacency matrix, we assume that

$$
b_{i j}=0 \Rightarrow m_{i j}(t)=0, \forall t \geq t_{0} .
$$


Moreover, we assume that for all $t \geq t_{0}, l(t) \geq \underline{l}>0$ similarly to the unperturbed case dealt with in Section IIIB. Then, it is always possible to find a $\varepsilon^{*}$ so that for all $\varepsilon \in\left[0, \varepsilon^{*}\right], a_{i j}(t, \varepsilon) \geq 0$. In the sequel, we assume that the perturbation parameter $\varepsilon$ satisfies this constraint. Finally, we further suppose the following important restriction.

Assumption 3: The perturbation on the interaction weights is bounded as follows :

$$
d_{\max } \triangleq \int_{t_{0}}^{\infty} m(t) d t<\infty .
$$

In matrix form, system (1) with interaction weights perturbed as in (14) rewrites as

$$
\left\{\begin{array}{l}
\dot{x}\left(t, \varepsilon, t_{0}\right)=-\left(l(t) L_{0}+\varepsilon M(t)\right) x\left(t, \varepsilon, t_{0}\right), \\
\text { with } x\left(t_{0}, \varepsilon, t_{0}\right)=x_{0} .
\end{array}\right.
$$

where matrix $M(t)$ is the Laplacian matrix associated to the adjacency matrix $\left(m_{i j}(t)\right)_{i j}$.

In the remaining of the section we study how $q\left(t_{1}, \varepsilon\right)$ behaves when the parameter $\varepsilon$ varies. In particular, we show that this variation is smooth, and that $q\left(t_{1}, \varepsilon\right) \underset{\varepsilon \rightarrow 0}{\longrightarrow} p_{0}$ as expected from Proposition 2. This is not obvious since it requires interchanging $\lim _{\varepsilon \rightarrow 0}$ and $\lim _{t \rightarrow \infty}$. We will use the definition of $q\left(t_{1}, \varepsilon\right)$ in function of the fundamental matrix $\Phi\left(t, \varepsilon, t_{1}\right): \mathbf{1}_{n} q\left(t_{1}, \varepsilon\right)^{T}=\lim _{t \rightarrow \infty} \Phi\left(t, \varepsilon, t_{1}\right)$. To show the smoothness of $q\left(t_{1}, \varepsilon\right)$ w.r.t $\varepsilon$, it suffices to show the smoothness of $\lim _{t \rightarrow \infty} \Phi\left(t, \varepsilon, t_{1}\right)$ w.r.t. $\varepsilon$. For this, we will turn to theorems allowing to interchange limits and differentiation.

First, notice that by Lemma 2 the fundamental matrix $\Phi\left(t, \varepsilon, t_{1}\right)$ described by system (7) in the particular case of system (15) satisfies

$$
\Phi\left(t, \varepsilon, t_{1}\right)=e^{-\tau\left(t, t_{1}\right) L_{0}}+R\left(t, \varepsilon, t_{1}\right)
$$

where

$$
\left\{\begin{array}{l}
R\left(t, \varepsilon, t_{1}\right)=\int_{t_{1}}^{t} G\left(s, \varepsilon, t_{1}\right) d s \\
G\left(s, \varepsilon, t_{1}\right)=e^{-\tau(t, s) L_{0}}(-\varepsilon) M(s) \Phi\left(s, \varepsilon, t_{1}\right),
\end{array}\right.
$$

and $\tau\left(t, t_{1}\right)=\int_{t_{1}}^{t} l(w) d w$. Also, denote

$$
R^{*}\left(t_{1}, \varepsilon\right)=\lim _{t \rightarrow \infty} R\left(t, \varepsilon, t_{1}\right) .
$$

The next lemma guarantees the existence of $R^{*}$.

Lemma 6: Under Assumption 3, function $R^{*}\left(t_{1}, \varepsilon\right)$ is continuously differentiable with regards to $\varepsilon$ for $\varepsilon \in I$ and

$$
\frac{\partial R^{*}}{\partial \varepsilon}\left(t_{1}, \varepsilon\right)=\int_{t_{1}}^{+\infty} \frac{\partial G}{\partial \varepsilon}\left(s, \varepsilon, t_{1}\right) d s,
$$

where

$$
\left\|\frac{\partial G}{\partial \varepsilon}\left(s, \varepsilon, t_{1}\right)\right\|_{\infty} \leq\|M(s)\|_{\infty}\left(1+n \varepsilon^{*}\|M\|\right) .
$$

The proof of the previous lemma is rather technical and is provided in Appendix B in [15]. A consequence of the previous result is the smoothness of $q\left(t_{1}, \varepsilon\right)$ with regards to $\varepsilon:$
Theorem 2: Under Assumption 3, both $q\left(t_{1}, \varepsilon\right)$ and $R^{*}\left(t_{1}, \varepsilon\right)$ are continuously differentiable in $\varepsilon$ for all $t_{1}$ and

$$
q\left(t_{1}, \varepsilon\right)=p_{0}+\frac{1}{n}\left(R^{*}\left(t_{1}, \varepsilon\right)\right)^{T} \mathbf{1}_{n} .
$$

Proof: According to Proposition 2, we have

$$
\lim _{t \rightarrow \infty} \Phi\left(t, \varepsilon, t_{1}\right)=\mathbf{1}_{n} p_{0}^{T}+R^{*}\left(t_{1}, \varepsilon\right),
$$

where $p_{0}$ is independent both of $t_{1}$ and $\varepsilon$. By definition of $q\left(t_{1}, \varepsilon\right)$, we have $\mathbf{1}_{n} q(t, \varepsilon)^{T}=\mathbf{1}_{n} p_{0}^{T}+R^{*}\left(t_{1}, \varepsilon\right)$. By transposing and right-multiplying by $\mathbf{1}_{n}^{T} / n$ provides

$$
q\left(t_{1}, \varepsilon\right)=p_{0}+\frac{1}{n}\left(R^{*}\left(t_{1}, \varepsilon\right)\right)^{T} \mathbf{1}_{n} .
$$

From Lemma $6, R^{*}\left(t_{1}, \varepsilon\right)$ is also continuously differentiable with regards to $\varepsilon$ for $\varepsilon \in I$ and so is $q\left(t_{1}, \varepsilon\right)$

One interesting corollary to Lemma 6 and Theorem 2 is that $q(t, \varepsilon)$ converges as $t \rightarrow+\infty$ :

Corollary 2: Suppose that Assumption 3 is satisfied. Then, we have

$$
\left\|q\left(t_{1}, \varepsilon\right)-p_{0}\right\| \leq \frac{\varepsilon}{n} \int_{t_{1}}^{+\infty}\|M(s)\|_{\infty} d s,
$$

and in particular, $q\left(t_{1}, \varepsilon\right)$ converges to $p_{0}$ as $t_{1} \rightarrow+\infty$ even for positive $\varepsilon$.

Proof: First notice that by Lemma $6, R^{*}$ exists and

$$
R^{*}\left(t_{1}, \varepsilon\right)=-\varepsilon \int_{t_{1}}^{\infty} e^{-\tau(t, s) L_{0}} M(s) \Phi\left(s, \varepsilon, t_{1}\right) d s .
$$

By equations (8) and (12), both $\left\|\Phi\left(s, \varepsilon, t_{1}\right)\right\|_{\infty}=1$ and $\left\|e^{-\tau(t, s) L_{0}}\right\|=1$. Then, we can use the triangular inequality to reverse integral and norm and conclude using that $\|\cdot\|_{\infty}$ is a submultiplicative norm to obtain

$$
\left\|R^{*}\left(t_{1}, \varepsilon\right)\right\| \leq \varepsilon \int_{t_{1}}^{\infty}\|M(s)\|_{\infty} d s .
$$

Theorem 2 allows to conclude. Finally, since $M \in \mathcal{L}_{1}$, the previous norm converges to 0 when $t_{1} \rightarrow \infty$.

It is noteworthy that Corollary 2 ensures that $q\left(t_{1}, \varepsilon\right)$ converges toward $p_{0}$ when either $\varepsilon \rightarrow 0$ or $t_{1} \rightarrow \infty$. For the later it is important to integrate $\|M(s)\|_{\infty}$ on the interval $\left[t_{1}, \infty\right)$ in (17) although the inequality would still hold replacing $t_{1}$ by $t_{0}$. The convergence of $q$ given in the previous corollary may seem natural. However, it is not always true for general consensus systems of type (5), even in instances where the systems converges to consensus. This fact is illustrated via an example in Section IV-B.

Corollary 2 can be used to study the evolution of $q$ in a faster time scale than the one given by time $t$. In particular, one may then study the limit of $q(t / \varepsilon, \varepsilon)$ when $\varepsilon \rightarrow 0$. However, this is out of the scope of the present paper.

\section{B. Example of oscillating $q$}

The aim of this section is to show that systems which involve uniformly varying weights with non vanishing perturbation, unlike the ones described in Section IV-A, may lead to a non converging vector $q$. Let us study a system of 

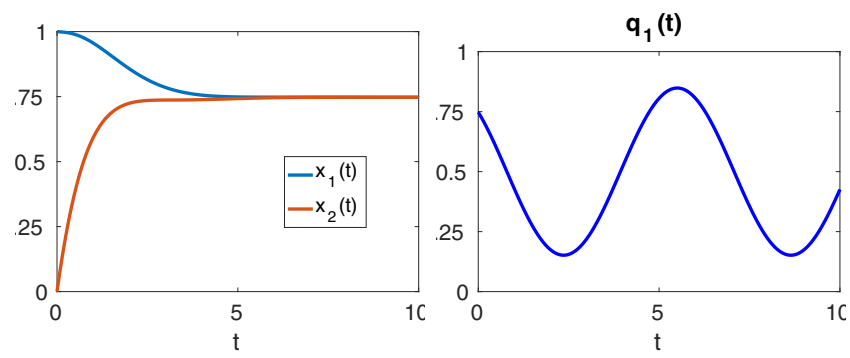

Fig. 1. (Left) trajectory of 2-agent system (1) for interaction weights defined in equation (18) with $\varepsilon=0.9$. (Right) first component $q_{1}(t, \varepsilon)$ of the convex combination providing the asymptotic consensus value $\alpha$ in function of the current state $x(t, \varepsilon, 0)$.

type (1) with two agents. The interaction weights have the following evolution in time

$$
\left\{\begin{array}{l}
a_{12}(t, \varepsilon)=\frac{1}{2}(1+\varepsilon \cos (t)), \\
a_{21}(t, \varepsilon)=\frac{1}{2}(1-\varepsilon \cos (t)),
\end{array}\right.
$$

where $\varepsilon \in[0,1)$ is a constant parameter. This system satisfies Assumption 1 with finite $K_{I}(\varepsilon)=\frac{1+\varepsilon}{1-\varepsilon} \geq 1$, and as a consequence converges to consensus (see Proposition 1). However, the system does not satify Assumption 3, required for Corollary 2 to apply. Setting $x_{1}(0)=1$ and $x_{2}(0)=0$ and integrating equation (1), we can show that

$$
x_{1}(t, \varepsilon, 0)-x_{2}(t, \varepsilon, 0)=e^{-t},
$$

from which, by integration, exact solutions for $x_{1}$ and $x_{2}$ can be obtained as :

$$
x_{1}(t, \varepsilon, 0)=\alpha(0, \varepsilon)+e^{-t} \frac{\varepsilon(\sin (t)-\cos (t))+2}{4},
$$

where the final consensus value is

$$
\alpha(0, \varepsilon)=\frac{\varepsilon}{4}+\frac{1}{2} .
$$

For this case, the convex combination $q$ can be obtained using $q_{1}+q_{2}=1$ and

$$
\begin{aligned}
q_{1}(t, \varepsilon) & =1-\frac{x_{1}\left(t, \varepsilon, t_{0}\right)-\alpha(0, \varepsilon)}{e^{-t}} \\
& =\frac{\varepsilon(\cos (t)-\sin (t))+2}{4}
\end{aligned}
$$

which does not converge despite asymptotic consensus for $x_{1}$ and $x_{2}$ as long as $\varepsilon \neq 0$ as shown in Figure 1 : the contribution of $x_{1}$ and $x_{2}$ for the asymptotic consensus value keeps oscillating over time.

\section{CONCLUSION}

In this paper, we have studied linear consensus systems with directed and time-varying interactions. The focus was on characterizing the asymptotic consensus value of the system. We have shown that this consensus value can be expressed as a convex combination of the current state. Under the cut-balance assumption on the interaction weights, the components of the convex combination have been nontrivially bounded. This means that each agent plays a non vanishing role in the asymptotic consensus value. When the interaction weights vary uniformly throughout the network, the convex combination is constant in time. We have also shown that when the uniformly varying interaction weights are perturbed by a $\mathcal{L}_{1}$ perturbation, the convex combination changes smoothly with regards to the perturbation parameter $\varepsilon$. Finally, we have demonstrated that for such kind of perturbation, the convex combination converges asymptotically in time. On the contrary, when this assumption is not satisfied, we have provided a case where the convex combination keeps oscillating over time while the agents converge to a consensus.

On-going works include applying the present results to time-scale separation in consensus systems.

\section{REFERENCES}

[1] L. Moreau, "Stability of multiagent systems with time-dependent communication links," IEEE Trans. on Automatic Control, vol. 50, no. 2, pp. 169-182, 2005.

[2] W. Michiels and H. Nijmeijer, "Synchronization of delay-coupled nonlinear oscillators: An approach based on the stability analysis of synchronized equilibria," Chaos, vol. 19, 2009.

[3] I.-C. Morărescu, W. Michiels, and M. Jungers, "Effect of a distributed delay on relative stability of diffusely coupled systems, with application to synchronized equilibria," International Journal of Robust and Nonlinear Control, vol. 26, no. 7, pp. 1565-1582, 2016.

[4] R. Olfati-Saber and R. Murray, "Consensus problems in networks of agents with switching topology and time-delays," IEEE Trans. on Automatic Control, vol. 49, pp. 1520-1533, 2004.

[5] W. Ren and R. W. Beard, "Consensus seeking in multiagent systems under dynamically changing interaction topologies," IEEE Trans. on Automatic Control, vol. 50, no. 5, pp. 655-661, 2005.

[6] J. M. Hendrickx and J. N. Tsitsiklis, "Convergence of type-symmetric and cut-balanced consensus seeking systems." IEEE Trans. on Automatic Control, vol. 58, no. 1, pp. 214-218, 2013.

[7] S. Martin and A. Girard, "Continuous-time consensus under persistent connectivity and slow divergence of reciprocal interaction weights." SIAM Journal on Control and Optimization, vol. 51, no. 3, pp. 25682584, 2013.

[8] J. Tsitsiklis, "Problems in decentralized decision making and computation," Ph.D. dissertation, PhD Thesis,Massachusetts Institute of Technology, 1984.

[9] J. Tsitsiklis, D. Bertsekas, and M. Athans, "Distributed asynchronous deterministic and stochastic gradient optimization algorithms," IEEE Trans. on Automatic Control, vol. 31, pp. 803-812, 1986.

[10] I. C. Morărescu, S. Martin, A. Girard, and A. Muller-Gueudin, "Coordination in networks of linear impulsive agents," IEEE Trans. on Automatic Control, vol. 61, no. 9, pp. 2402-2415, Sept 2016.

[11] M. C. Bragagnolo, I. C. Morărescu, J. Daafouz, and P. Riedinger, "Reset strategy for consensus in networks of clusters." Automatica, vol. 65, pp. 53-63, 2016.

[12] A. F. Filippov, Differential equations with discontinuous righthand sides. Dordrecht: Kluwer Academic Publishers Group, 1988, translated from the Russian.

[13] V. I. Arnold, Ordinary differential equations, ser. Universitext. Berlin: Springer-Verlag, 2006, translated from the Russian by Roger Cooke, Second printing of the 1992 edition.

[14] C. W. Wu, "On rayleigh-ritz ratios of a generalized laplacian matrix of directed graphs," Linear algebra and its applications, vol. 402, pp. 207-227, 2005.

[15] S. Martin, I.-C. Morarescu, and D. Nesic, "Consensus value estimates in time-varying and directed networks," http://w3.cran.univlorraine.fr/perso/samuel.martin/publications/CDC2017.pdf, 2017.

[16] S. Martin and J. M. Hendrickx, "Continuous-time consensus under non-instantaneous reciprocity," IEEE Trans. on Automatic Control, vol. 61, no. 9, pp. 2484-2495, Sept 2016. 